\title{
Attraktivität hat ihren Preis: hohe reale Wohnkosten, weites Pendeln oder lange Warteschlangen
}

\author{
Matthias Wrede
}

Online publiziert: 4. November 2019

(C) Der/die Autor(en) 2019

Zusammenfassung Höhere Mieten bzw. Immobilienpreise und/oder niedrige Löhne werden auf funktionierenden Märkten die höhere natürliche Standortattraktivität einer Region bzw. ein attraktiveres Angebot öffentlicher Güter ausgleichen, wenn die Menschen Wohn- und Arbeitsort frei wählen können. Wenn Lohn- und Mietenunterschiede zwischen lokalen Wohnungs- und Arbeitsmärkten durch staatliche Preisregulierung verhindert bzw. reduziert werden, dann führt das in einigen Regionen zu Nachfrage- und in anderen Regionen zu Angebotsüberschüssen. Wanderungsbewegungen, die Versorgungsengpässe verstärken können, werden ausgelöst. Auf Märkten mit Friktionen allerdings haben neben Preisen auch Mengen eine kompensatorische Funktion. Längere Suchzeiten auf lokalen Wohnungs- und Arbeitsmärkten wirken ebenfalls den Anziehungskräften attraktiver Standorte entgegen. Dieser Aufsatz stellt die grundlegenden Mechanismen dar und erläutert diese anhand stilisierter Fakten.

Schlüsselwörter Regionale Lebensqualität · Kompensatorische Differentiale · Suchfriktionen

\section{The cost of attractiveness: Higher rents, further commutes, and longer queues}

Abstract Higher rents or real estate prices and / or low wages will compensate for the higher natural attractiveness of a region or a more attractive supply of public goods in functioning markets, if people are free to choose their place of residence

\footnotetext{
M. Wrede $(\triangle)$

Fachbereich Wirtschafts- und Sozialwissenschaften, Friedrich-Alexander-Universität

Erlangen-Nürnberg (FAU), Postfach 3931, 90020 Nürnberg, Deutschland

E-Mail: matthias.wrede@fau.de

CESifo, München, Deutschland
} 
and work. If wage and rent differences between local housing and labor markets are eliminated or reduced by state price regulation, this leads to excess demand in some regions and to excess supply in other regions. Migration that can increase supply shortages is triggered. In markets with frictions, however, in addition to prices, quantities also have a compensatory function Long search times on local housing and labor markets also counteract the appeal of attractive locations. This review presents the basic mechanisms and explains them using stylized facts.

Keywords Regional quality of life - Compensating differentials · Search frictions

\section{Einleitung}

Regionale Immobilien- und Arbeitsmärkte sind komplexe interdependente Systeme, in denen nicht nur die Märkte innerhalb einer Region interagieren, sondern auch die Märkte über die Regionsgrenzen hinweg. Die Interdependenz ist darin begründet, dass Wohnen und Arbeiten an Standorte gebunden sind und die Überwindung von Raum mit Kosten verbunden ist. Menschen können sowohl ihren Arbeits- als auch ihren Wohnort verlagern und von ihrem Wohnort zu einem erreichbaren Arbeitsort pendeln. Zugleich ist nicht nur Wohnen, sondern auch Arbeiten für die meisten Menschen essentiell, da sie ihren Lebensunterhalt einschließlich des Wohnens mit Arbeitseinkommen finanzieren müssen.

Die Interdependenz der Märkte hat zur Folge, dass regulierende Eingriffe des Staates Konsequenzen für andere Märkte und Regionen haben. Dafür lassen sich beliebig viele Beispiele finden: Sozialer Wohnungsbau in den Städten kann Zuwanderung Begünstigter verstärken und Abwanderung verringern und damit in anderen Regionen das Arbeitsangebot und die Nachfrage nach Wohnraum reduzieren. Obergrenzen für Mieten können die Konkurrenz auf dem Mietwohnungsmarkt für zahlungskräftigere Haushalte erhöhen und diese in den Markt für Wohneigentum und/ oder das nicht-regulierte Umland abdrängen. Mindestlöhne können die Konkurrenzfähigkeit weniger produktiver Regionen in Branchen, die weitgehend friktionslos handelbare Güter herstellen, untergraben. Die Interdependenz der Märkte bewirkt zudem, dass Preis- und Mengenregulierung auf einem Teil der Märkte kompensierende Preis- und Mengenregulierung auf anderen Märkten und gegebenenfalls eine Regulierungsspirale auslösen können, welche die Komplexität und die Kosten der Regulierung erhöht sowie deren Passgenauigkeit untergräbt.

Aufgrund der Unverzichtbarkeit von Wohnen und Arbeiten sind regulierende Eingriffe des Staates und deren Folgen jedoch wirtschaftlich und gesellschaftlich bedeutsam und müssen daher besonders sorgfältig geplant und vorbedacht werden. Dazu ist eine genaue Kenntnis und ein gutes Verständnis der Märkte, der Institutionen und der relevanten empirischen Parameter erforderlich.

Dieser Aufsatz soll grundlegend Mechanismen der interdependenten Systeme regionaler Immobilien- und Arbeitsmärkte darstellen. Die Betrachtung beschränkt sich nicht auf den Referenzfall vollkommener Märkte, sondern bedenkt Suchfriktionen in beiden Marktkategorien mit. Anhand stilisierter Fakten werden die Zusammenhänge für deutsche Landkreise und kreisfreie Städte illustriert. Auf eine vollständige 
Simulation des Gesamtsystems wird dabei allerdings verzichtet. Implikationen für Preisregulierungen auf den Wohnungsmärkten werden im Zuge der Darstellung erörtert.

\section{Räumliches Gleichgewicht und Standortfaktoren}

Da Boden für Haushalte und Unternehmen als physische Grundlage des Aufenthalts, des Konsums, der Produktion und des Transports von Gütern und Dienstleistungen unverzichtbar ist, müssen alle Wirtschaftssubjekte einen Standort für ihre wirtschaftlichen Aktivitäten wählen. Die Wahl des Standorts ist aufgrund bestehender Raumüberwindungskosten nicht beliebig. Da der Transport von Gütern, Dienstleistungen und Menschen mit Kosten verbunden ist und für einzelne Kategorien aus physikalischen Gründen räumlich sogar strikt limitiert ist, sind nicht alle Güter und Dienstleistungen zu gleichen Konditionen an allen Standorten verfügbar. Die meisten Individuen und Firmen sind bereit, den Standort zu verlagern, wenn andere Standorte deutlich bessere Möglichkeiten für die wirtschaftliche und persönliche Entfaltung bieten. Die Verlagerung des Standorts verursacht allerdings zum Teil erhebliche Transaktionskosten, da Informationen über Alternativen beschafft und bewertet, Gegenstände zum neuen Standort transportiert und soziale Beziehungen neu gestaltet werden müssen. Standorte werden nur dann verlagert, wenn die dadurch erreichte Verbesserung die Transaktionskosten übersteigt. Da relative Standortvorteile und Transaktionskosten sich zwischen den einzelnen Individuen und Firmen unterscheiden, werden nicht alle auf dieselbe Weise auf Standortunterschiede reagieren, aber wachsende Unterschiede werden zunehmende Wanderung bewirken.

Obwohl es sogar über längere Zeit zu erheblicher Zuwanderung in von vielen als attraktiv wahrgenommene Gebiete kommen kann, sind der Zuwanderung von Haushalten und Unternehmen vorübergehend und dauerhaft Grenzen gesetzt. Kurzfristig ist das Wohnungsangebot starr, sind die für Wohnungsbau und Gewerbe vorgesehenen Flächen sowie die benötigte physische und soziale Infrastruktur nicht vermehrbar. Langfristig können zusätzliche Flächen ausgewiesen werden, kann die Grundfläche dichter und höher bebaut werden, kann zusätzliche Infrastruktur bereitgestellt werden. Diese Anpassungsprozesse benötigen Zeit, verschlingen Ressourcen und können aus physikalischen Gründen auch nicht beliebig fortgesetzt werden. Die Wanderungsprozesse lösen, ohne dass es dazu staatlicher Lenkung bedarf, Veränderungen aus, die Zuwanderung in Zielregionen von Wanderung verlangsamen und schließlich zum Stillstand bringen. Zum einen führt die Zuwanderung von Haushalten und Firmen zu größerer Nutzungsintensität des Bodens und der Infrastruktur bzw. anderer öffentlicher Leistungen. Überfüllung setzt ein; Fahrtzeiten und Wartezeiten nehmen zu. Auch Lärmbelastung sowie Luft- und Wasserverschmutzung werden intensiver bzw. erfordern größere Vermeidungsaufwendungen. Die Attraktivität des Standorts wird durch Ballungskosten reduziert. Obwohl eine höhere Dichte von Beschäftigten und Firmen, die Auswahl vergrößert und ein besseres Matching 
ermöglicht (Dauth et al. 2018)1, erhöht die zunehmende Komplexität der Märkte die Kosten der Suche. Auf den Wohnungsmärkten dauert es länger, eine passende Wohnung zu finden. Wohnungsanbieter haben bei hoher zahlungskräftiger und -williger Nachfrage die Möglichkeit, Marktmacht auszuüben und Mieter präferenzbasiert auszuwählen. Die Individuen müssen ihre Suche nach einer Wohnung auf ein immer größeres Gebiet ausdehnen und längere Fahrtzeiten und Wege zum Arbeitsplatz in Kauf nehmen. Der Attraktivität des Standorts wirken bei anhaltendem Zuwanderungsdruck jedoch nicht nur die zunehmenden realen Kosten der Ballung entgegen, sondern auch sich verändernde Preise. Ein Nachfrageüberhang auf Miet-, Eigentumswohnungs- und Bodenmärkten führt zu steigenden Preisen. Vorbehaltlich entgegenstehender Regulierung steigen zunächst Wieder- und Neuvertragsmieten sowie Eigentumwohnungspreise und mit Verzögerung Baulandpreise und Bestandsmieten. Ähnlich verhält es sich bei den Märkten für Gewerbeimmobilien und -flächen; auch deren Preise steigen. Diese Preiserhöhungen senken für Haushalte und Unternehmen die Standortattraktivität. Produktivere Unternehmen können zwar höhere Löhne zahlen und damit einen Teil des Anstiegs lokaler Preise ausgleichen, doch die Löhne lassen sich nur soweit steigern, wie es durch eine größere (Grenz-) Produktivität der Arbeit gerechtfertigt ist; aus Kostengründen können Unternehmen diesen Prozess nicht beliebig fortsetzen.

Letztlich werden Standortverlagerungen von Haushalten und Unternehmen zum Erliegen kommen. Natürliche und durch Agglomerationsvorteile bedingte Standortvorteile einzelner Regionen, die ein höheres Lohnniveau bewirken, werden durch höhere Ballungskosten und längere Fahrt- und Suchzeiten sowie höhere Mieten, Wohnungs-, Gewerbeimmobilien- und Bodenpreise ausgeglichen. Anders ausgedrückt: In einem räumlichen Gleichgewicht, in dem zentrifugale und zentripetale Kräfte einander ausgleichen, werden Individuen und Firmen in dünner besiedelten peripheren Lagen durch geringere Ballungskosten und niedrigere mit der Bodennutzung verbundene Preise für geringere Produktivität und Löhne entschädigt. Bei vorhandener Mobilität werden zumindest die marginalen Akteure, die nicht stark an eine Region gebunden sind, in den verschiedenen Regionen gleichgestellt. Unter Berücksichtigung aller Einflussfaktoren - wie natürliche und menschlich geschaffene Lebens- und Produktionsbedingungen sowie räumlich differenzierte Preise - lohnt sich für diese Individuen und Firmen ein Standortwechsel angesichts bestehender Transaktionskosten nicht. Bezogen auf diese marginalen Akteure ist das räumliche Gleichgewicht angesichts gleicher Nutzen auch gerecht. Obwohl starke Unterschiede zwischen den Regionen bei einzelnen Standortfaktoren bestehen können, ist aus der Sicht der mobilen Akteure in einer Gesamtschau kein relevanter Niveauunterschied zu erkennen. Gleiche erzielen in verschiedenen Regionen gleichen Nutzen, aber auf unterschiedliche Weise.

Solange freie Standortwahl für Haushalte und Firmen nicht per Regulierung ausgeschlossen wird, werden sich eigentlich lokal begrenzte politische Maßnahmen wie die Bereitstellung lokaler öffentlicher Güter oder auf bestimmte Regionen beschränkte (Preis-) Regulierungen auch auf andere Regionen auswirken. Wenn sich

\footnotetext{
1 Für einen Überblick zu den quantitativen Zusammenhängen von Bevölkerungsdichte und zahlreichen ökonomischen Größen siehe Ahlfeldt und Pietrostefani (2019).
} 
die relative Standortattraktivität ändert und lokale Preise reagieren, dann werden Wanderungsbewegungen ausgelöst, die wiederum in den Herkunftsregionen Auswirkungen auf die Produktions- und Lebensbedingungen sowie die lokalen Preise zeitigen. Durch Mobilität sind Märkte und soziale Systeme verschiedener Regionen miteinander verzahnt und müssen als komplexes Gesamtsystem betrachtet werden.

Aus diesen Überlegungen folgt unmittelbar, dass in einem ökonomischen System mit Konsumentensouveränität und freier Mobilität Preise nicht friktionslos handelbarer Güter nicht an allen Orten mit unterschiedlichen Merkmalen gleich hoch sein können und dass aus Preisunterschieden auf Unterschiede in den realen Standortbedingungen zurückgeschlossen werden kann. Der Wert, den Haushalte und Firmen den lokalen Standortbedingungen zumessen, kann aus den prinzipiell beobachtbaren Preisunterschieden präzise berechnet werden (Rosen 1979; Roback 1982). Bei der Beschreibung dieser Methode wird im Folgenden - der Einfachheit halber - von identischen interregional perfekt mobilen Individuen und Firmen ausgegangen, die jeweils eine Einheit Arbeit und Boden bereitstellen bzw. nutzen, um ein handelbares Gut herzustellen. Arbeit und Boden weisen keine Qualitätsunterschiede auf, GröBenvorteile in der Produktion seien ausgeschlossen. Zudem wird von Immobilien abstrahiert, so dass eine Einheit Boden mit einer Wohnung gleichgesetzt wird. Es sei außerdem angenommen, dass die lokale Lebensqualität und Produktivität von Regionen durch eine stetige Größe $A$ gemessen werden kann. In einem räumlichen Gleichgewicht bei vollständiger Konkurrenz müssen die Durchschnittskosten $C$ dem Preis des handelbaren Gutes $P$ und der lokal erzielte Nutzen $V$ dem außerhalb der Region erreichbaren Nutzen $\bar{V}$ entsprechen:

$$
\begin{aligned}
& V(W, R ; A)=\bar{V} \\
& C(W, R ; A)=P,
\end{aligned}
$$

wobei $W$ den Lohn und $R$ den Boden(nutzungs)preis angeben. Die Zahlungsbereitschaften für eine Erhöhung der lokalen Qualität von Individuen und Firmen $Z B_{\mathrm{I}}$ und $Z B_{F}$ lassen sich berechnen als

$$
Z B_{I}=\frac{d R}{d A}-\frac{d W}{d A} \quad \text { und } \quad Z B_{F}=\frac{d R}{d A}+\frac{d W}{d A},
$$

wobei $d R / d A$ und $d W / d A$ angeben, wie stark Bodenpreis und Lohn steigen, wenn die lokale Qualität sich erhöht. Je stärker der Bodenpreis mit der lokalen Qualität steigt und je stärker der Lohn mit der lokalen Qualität sinkt, desto höher muss die Zahlungsbereitschaft der Individuen sein. Die Zahlungsbereitschaft der Firmen muss um so höher ausfallen, je stärker Bodenpreis und Lohn mit der Qualität steigen. Wäre dem nicht so, würden Individuen bzw. Firmen ab- oder zuwandern. Aus den Zusammenhängen zwischen Eigenschaften der Regionen einerseits und Löhnen bzw. Bodenpreisen andererseits lassen sich diese Zahlungsbereitschaften für konkrete Eigenschaften und Regionen bestimmen (für Deutschland z. B. Büttner und Ebertz 2009). Wenn Individualdaten für Löhne und Grundstücke bzw. Immobilien vorhanden sind, können auch Zahlungsbereitschaften für das vorhandene Bündel an Standortfaktoren direkt ohne Erfassung der einzelnen regionalen Merkmale ermittelt werden (z.B. Albouy 2012). Der Ansatz kann so erweitert werden, dass 
vorhandenen Verzerrungen der Marktpreise Rechnung getragen wird. In Studien werden lokale öffentliche Finanzen (Gyourko und Tracy 1991), bundesweite Einkommensteuern (Albouy 2009, 2016), Pendeln (Albouy und Lue 2015), Unterschiede zwischen Mieten und Hauspreisen (Winters 2010) sowie Mobilitätskosten (Bayer et al. 2009) berücksichtigt. Innerhalb von Städten muss analog für eine geringere Entfernung zu Stadtzentrum, Arbeitsplätzen und Freizeit- und Kulturstätten mit höheren Bodenpreisen gezahlt werden (siehe beispielhaft für viele empirische Studien Ahlfeldt 2011; Ahlfeldt und Maennig 2010).

\section{Räumliches Gleichgewicht, Suchfriktionen und Standortfaktoren}

Aufgrund fehlender Informationen, bestehender Regulierung oder unflexibler Preise ist es nicht immer möglich, angestrebte Vertragsverhältnisse im gewünschten Umfang sofort einzugehen. Dies trifft insbesondere auf Märkte für komplexere oder aufwendig herzustellende Güter oder Dienstleistungen zu; auf Arbeits- und Wohnungsmärkten sind auch längere Suchphasen fast unvermeidbar. Geeignete Arbeitsplätze etwa müssen erst identifiziert und ausgewählt und Vertragskonditionen festgelegt werden. Dies hat zur Folge, dass insbesondere nach einer Kündigung Zeiten der Arbeitslosigkeit zu überstehen sind. Suchfriktionen wirken sich auf das individuelle Verhalten, aber auch auf Marktpreise und räumliche Strukturen aus. Es existiert inzwischen eine umfangreiche theoretische und empirische Literatur, die sich mit den Folgen von Suchfriktionen auf Wohnungs- und Arbeitsmärkten für den Marktzugang und die räumliche Verteilung verschiedener Gruppen auseinandersetzt (siehe für Arbeitsmärkte bzw. Immobilienmärkte Zenou 2009; Han und Strange 2015). Da viele Zusammenhänge allerdings sensitiv auf Änderungen der Suchmodalitäten, des Matching-Prozesses und kritischer Parameter reagieren (vgl. Duranton und Puga 2015), konzentriert sich die folgende Darstellung auf einige grundlegende Implikationen für die kompensatorische Funktion von Preisen und verzichtet auf eine differenzierte Analyse von Suchfriktionen und Institutionen auf durch solche Friktionen gekennzeichneten Märkten.

Wenn lokale Märkte Suchfriktionen aufweisen, dann sind neben den Preisen auch die erwarteten Vertragsdauern bzw. Suchzeiten für den Ausgleich der Nutzen über die Regionen hinweg ausschlaggebend. Harris und Todaro (1970) haben als eine der ersten am Beispiel der Wanderung aus ländlichen Regionen in Städte beschrieben, dass in einem räumlichen Gleichgewicht mit nach unten starren Löhnen in den Städten die Sucharbeitslosigkeit gerade so hoch ist, dass nicht die bei Beschäftigung realisierten Arbeitseinkünfte, sondern die ex-ante erwarteten Arbeitseinkünfte in Stadt und Land gleich hoch sind.

Wie Arbeitslosigkeit die Kompensationsfunktion von Löhnen und Immobilienpreisen bzw. Mieten verändert, lässt sich anhand eines einfachen Szenarios erkennen. Dazu wird davon ausgegangen, dass ein repräsentatives Individuum erwartet, mit einer bestimmten Wahrscheinlichkeit $p$ beschäftigt und andernfalls arbeitslos zu sein. Bei Arbeitslosigkeit werde Arbeitslosenunterstützung $B$ in festgelegter Höhe gewährt. Unterstellt man zudem - der Einfachheit halber - dass der Nutzen sich additiv aus dem Nutzen des Güterkonsums, des Wohnens und der lokalen Quali- 
tät zusammensetzt und der Grenznutzen des Einkommens konstant ist, dann kann die Zahlungsbereitschaft für eine Erhöhung der lokalen Qualität des repräsentativen Individuums $Z B_{I}^{A}$ dargestellt werden als

$$
Z B_{I}^{A}=\frac{d R}{d A}-p \frac{d W}{d A}-(W-B) \frac{d p}{d A},
$$

wobei $d p / d A$ beschreibt, wie sich die Wahrscheinlichkeit verändert, beschäftigt zu sein, wenn die lokale Qualität steigt. Da das betrachtete Individuum nicht erwartet, ununterbrochen beschäftigt zu sein, wird der Lohn mit dem erwarteten Anteil der Beschäftigungszeit $p$ gewichtet, wohingegen die Beschäftigungschancen mit dem Lohnabstand $W-B$ gewichtet werden. Je stärker die Wahrscheinlichkeit, beschäftigt zu sein, mit zunehmender lokaler Qualität sinkt, desto größer ist die Zahlungsbereitschaft für die lokale Qualität. Wrede (2015) hat gezeigt, dass ein vergleichbares Ergebnis auch in einem makroökonomischen Search-and-MatchingModell mit Lohnverhandlungen zwischen Arbeitnehmer und Arbeitgeber und endogenem Arbeitslosigkeitsniveau erzielt werden kann, das es für empirische Analysen erlaubt, relevante Parameter zur Berechnung der Beschäftigungswahrscheinlichkeit zu identifizieren.

Friktionen auf städtischen Wohnungsmärkten haben vergleichbare Auswirkungen. Betrachtet werde eine Metropolregion bestehend aus einer Stadt und dem Umland; wobei sich alle Arbeitsplätze in der Stadt befinden. Die Pendelkosten zwischen Umland und Stadt seien gegeben. Es sei angenommen, dass das repräsentative Individuum, das einen Umzug in die Metropolregion erwägt, erwartet, mit gegebener Wahrscheinlichkeit $q$ in der Stadt zu wohnen und andernfalls im Umland eine Wohnung nehmen zu müssen. Das Wohnungsangebot in der Stadt sei nicht variabel, das Angebot im Umland hingegen vollständig flexibel. In der Stadt wird jede verfügbare Bodenfläche genutzt, im Umland wäre hingegen Boden für weitere Wohnbebauung vorhanden. Jetzt sei unterstellt, dass die Boden- bzw. Immobilienpreise so strikt reguliert seien, dass es nicht zu einem Nutzenausgleich zwischen Stadt und Umland sowie zwischen Stadt und anderen Regionen kommt. Bei nach unten starren Preisen in der Stadt muss im räumlichen Gleichgewicht die Gesamtbevölkerung der Metropolregion gerade so hoch sein, das der erwartete Nutzen in der Metropolregion gleich dem Nutzen in alternativen Regionen ist:

$$
q V_{S}+(1-q) V_{U}=\bar{V}
$$

wobei $V_{S}$ den Nutzen in der Stadt und $V_{U}$ denjenigen im Umland angibt. Erneut sei unterstellt, dass der Nutzen additiv ist. Zudem wird angenommen, dass die lokale Qualität $A$ in der Stadt einen höheren Nutzen stiftet als im Umland. Die Zahlungsbereitschaft für eine Erhöhung der lokalen Qualität des repräsentativen Individuums $Z B_{I}^{W}$ ergibt sich dann als

$$
Z B_{I}^{W}=q \frac{d R_{S}}{d A}-\frac{d W}{d A}-\left(V_{S}-V_{U}\right) \frac{d q}{d A} .
$$

Dabei bezeichnet $R_{S}$ den Bodenpreis in der Stadt. Es wird angenommen, dass aufgrund des „Überangebots“ an Boden der Bodenpreis im Umland nicht auf Zu- und 

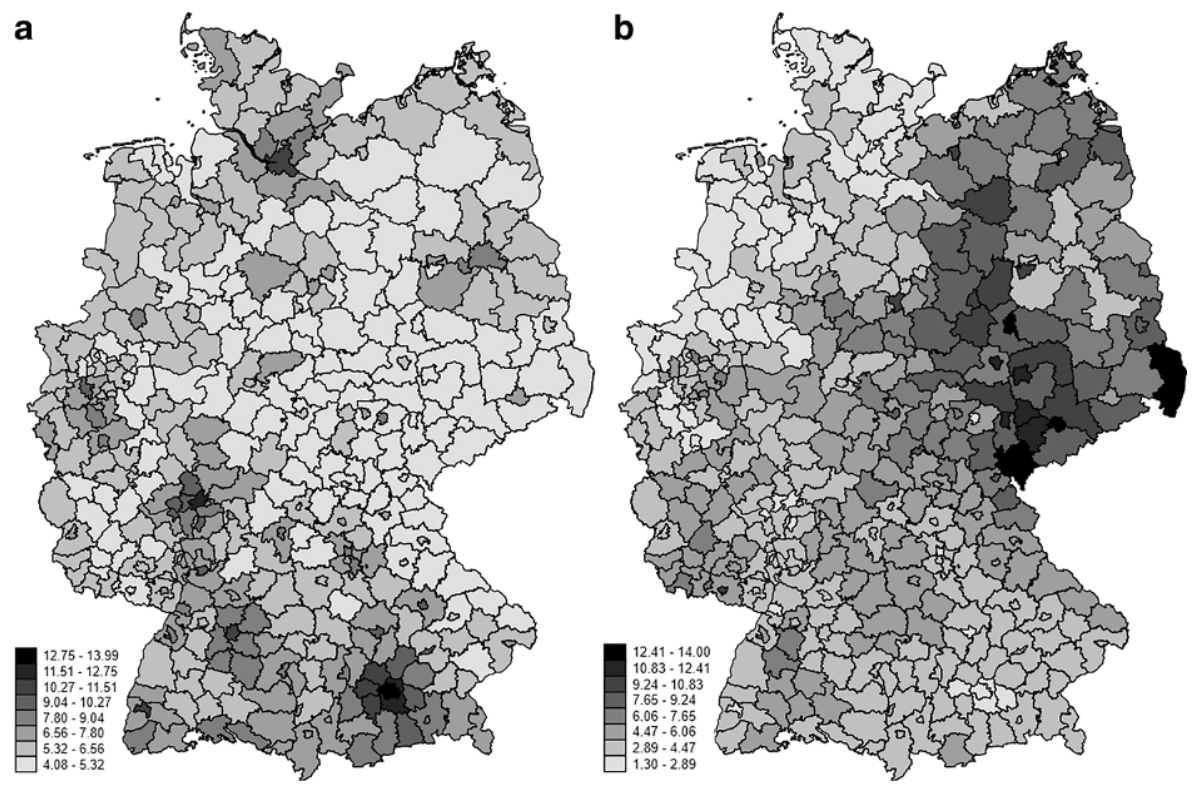

Abb. 1 a Angebotsmieten 2014 ( $€$ pro qm) b und Leerstandsquoten 2011 (in \%). Die Angebotsmieten entstammen der Wohnungsmarktbeobachtung des Bundesinstituts für Bau-, Stadt- und Raumforschung (BBSR). Die Wohnungsleerstände sind im Rahmen des Zensus 2011 vom Statistischen Bundesamt ermittelt worden

Abwanderung reagiert. ${ }^{2}$ Der Bodenpreis in der Stadt wird mit der Wahrscheinlichkeit, in der Stadt wohnen zu können, gewichtet, wohingegen die Chancen, eine Wohnung in der Stadt zu finden, mit dem Nutzenabstand für das Wohnen in Stadt und Umland $V_{S}-V_{U}$ gewichtet werden. Je stärker der Bodenpreis in der Stadt und je weniger stark der Lohn mit der lokalen Qualität steigt, desto höher ist die Zahlungsbereitschaft. Je mehr die Wahrscheinlichkeit, in der Stadt statt im Umland zu wohnen, mit zunehmender lokaler Qualität sinkt, desto größer ist bei der angenommenen Attraktivität des Stadtlebens die Zahlungsbereitschaft für die lokale Qualität. Aus einem solchen einfachen Modell lassen sich auch die Konsequenzen einer Preisregulierung für die Bevölkerungsströme ermitteln. Die Wahrscheinlichkeit, in der Stadt zu wohnen, gleicht deren Bevölkerungsanteil an der Metropolregion: $q=N_{S} /\left(N_{S}+N_{U}\right)$. Aufgrund der Annahme eines völlig starren Wohnungsangebots in der Stadt kann nur die Bevölkerung im Umland auf eine Veränderung der Preisregulierung in der Stadt reagieren:

$$
\frac{d N_{U}}{d R_{S}}=-\frac{N_{S}}{q\left(V_{S}-V_{U}\right)}<0 .
$$

\footnotetext{
2 Das Modell und damit die Formel für die Zahlungsbereitschaft kann um vielfältige Facetten (Immobilienmarkt, variierende Preise im Umland, Überfüllung auf Pendelstrecken, etc.) erweitert werden, ohne dass sich die Struktur der Formel für die Zahlungsbereitschaft fundamental ändern würde.
} 

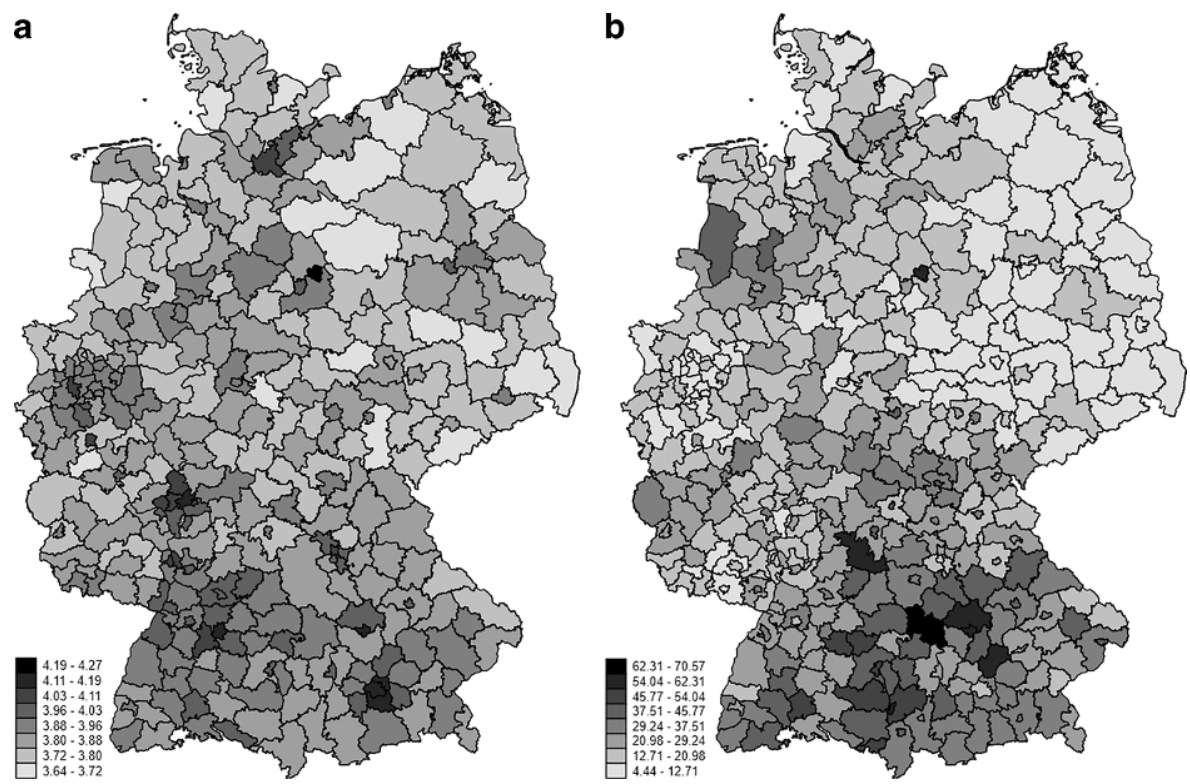

Abb. 2 a Regionales Lohnniveau 2014 (logarithmiertes adjustiertes Tagesentgelt) und b offene Stellen pro Arbeitslosem 2014 in \%. Die regionalen Lohnniveaus (für Löhne bis zur Beitragsbemessungsgrenze für die gesetzliche Rentenversicherung) werden für Kreisregionen aus anonymisierten Individualdaten gewonnen und mit Hilfe von hedonischen Lohnregressionen um Einflüsse des Geschlechts, des Alters, der Beschäftigungsdauer und der Ausbildung bereinigt. Datengrundlage ist der Scientific Use File SIAB-R bereitgestellt vom Forschungsdatenzentrum des IAB (Ganzer et al. 2016). Die Daten zu offenen Stellen und Arbeitslosigkeit stammen aus der Statistik der Bundesagentur für Arbeit

Wird der Bodenpreis in der Stadt aufgrund verschärfter Preisregulierung gesenkt, dann nimmt die Bevölkerung im Umland der Stadt zu. Die Bevölkerung der Metropolregion insgesamt steigt, aber ein zunehmender Anteil der Bevölkerung findet keine Wohnung in der Stadt. Die striktere Preisregulierung führt in den Metropolregionen $\mathrm{zu}$ einem Anstieg der Bevölkerung im Einzugsbereich der Beschäftigungszentren. ${ }^{3}$ Insoweit das Angebot in den Städten variabel ist, wird die Regulierung von Boden- und Immobilienpreisen das Wohnungsangebot und die Bevölkerungsgröße der Städte verändern. Eine striktere Preisregulierung auf dem Wohnungsmarkt wird alternative Bodennutzungen begünstigen und damit die Aufnahmefähigkeit der Städte verringern. Weniger zahlungskräftige Haushalte erhalten Zugang zu städtischen Wohnungsmärkten; wohlhabendere Haushalte werden folglich teilweise verdrängt. Wenn der Preismechanismus daran gehindert wird, über die Verwendung knapper Ressourcen zu entscheiden, dann treten andere Mechanismen wie politisch bestimmte Priorisierungen, Warteschlangen oder persönliche Beziehungen an dessen Stelle. Korruption bei Wohnungsgesellschaften und Steuer-

\footnotetext{
3 Die Preis- und Mengeneffekte der Wohnungsmarktregulierung hängen allerdings von der Spezifikation der Regulierung ab. Mense et al. (2019) etwa zeigen, dass die Ausnahmeregelung für Neubauten bei der Mietpreisbremse zur Folge hat, dass mehr zahlungskräftige Wohnungssuchende in regulierte Städte streben und die Nachfrage nach Bauland dort zunimmt.
} 
Abb. 3 a, b Regionales Lohnniveau und andere Parameter

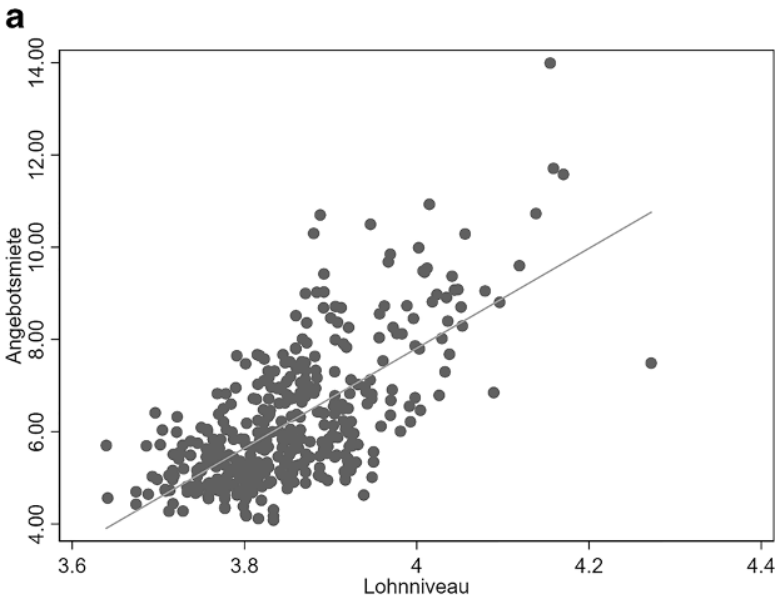

b

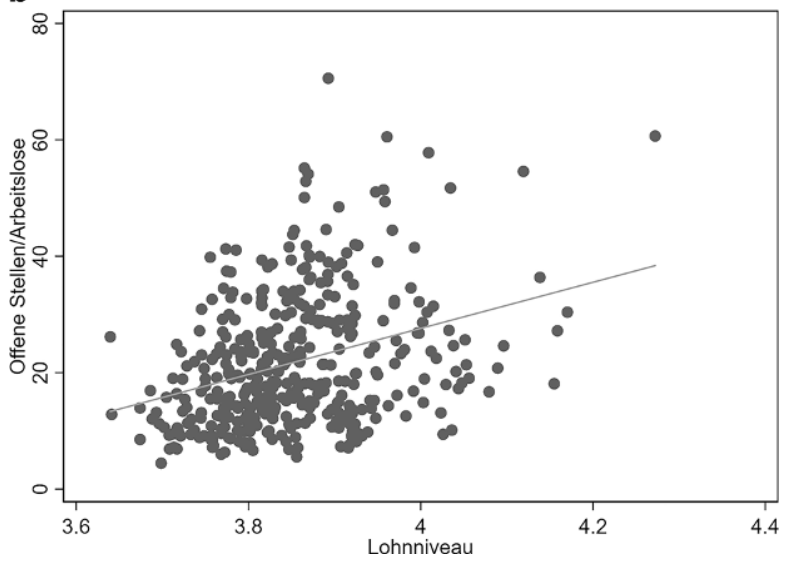

hinterziehung von nicht dokumentierten Geldleistungen sind die Folge. Vermieter erhalten den Spielraum, präferenzbasiert zu diskriminieren. Allokationsmechanismen, die nicht allein auf Preisen beruhen, verschlingen Ressourcen, reagieren weniger flexibel als der Preismechanismus auf Änderungen der ökonomischen und sozialen Verhältnisse, begünstigen Verschwendung bzw. haben unerwünschte Verteilungswirkungen.

\section{Lohnniveau, Lebenshaltungskosten, Matching in Deutschland}

Die dargestellten Überlegungen lassen erwarten, dass Preise und Friktionen auf Arbeits- und Immobilienmärkten in Deutschland über die Regionen variieren und dass diese Variation systematisch mit Unterschieden in Produktions- und Lebensbedingungen verknüpft ist. Die Analyse dieses Sachverhalts für Deutschland wird jedoch durch fehlende bzw. nicht-verfügbare Daten erschwert. Während Arbeitslosigkeit, offene Stellen und Löhne mit Daten der Bundesagentur für Arbeit bzw. des 
Abb. 4 a, b Angebotsmieten und andere Parameter

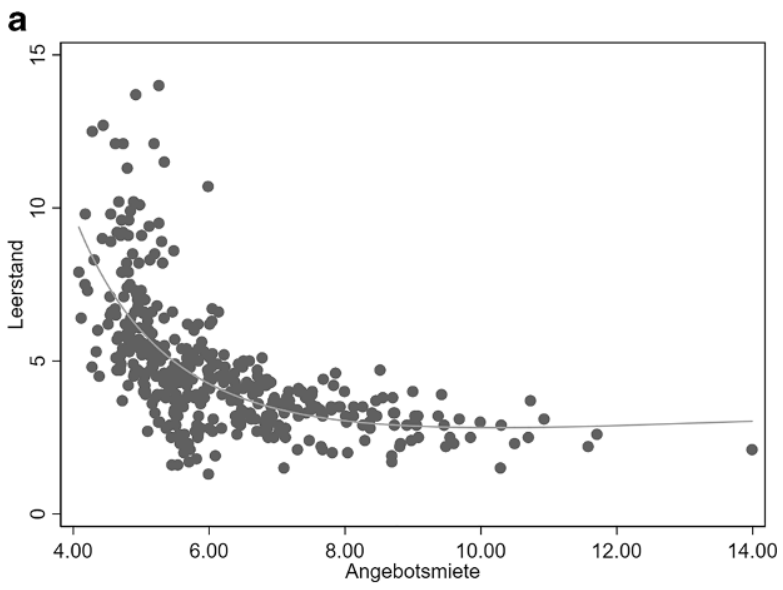

b

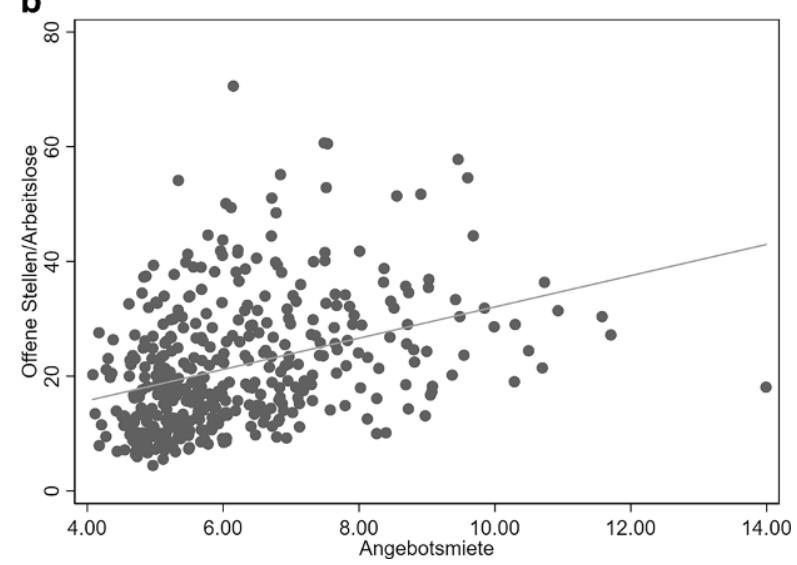

Instituts für Arbeitsmarkt- und Berufsforschung (IAB) auch auf Kreisebene im Zeitablauf genau dargestellt werden können, werden Daten über Immobilienmärkte und Lebenshaltungskosten nicht systematisch erfasst bzw. zur Verfügung gestellt. Für Wohnungsleerstände muss auf in großen Abständen durchgeführte Volkszählungen bzw. Fortschreibungen zurückgegriffen werden. Lebenshaltungskosten werden nicht umfassend kleinräumig erfasst und publiziert (eine Ausnahme ist die Analyse regionaler Daten für 2009 durch BBSR 2009). Zwar liegen den Gutachterausschüssen detaillierte Daten über Transaktionen auf den Immobilienmärkten vor, diese aber werden - zulasten einer informierten gesellschaftliche Debatte - nicht bundesweit einheitlich erfasst und veröffentlicht. Aus Immobilienportalen lassen sich zwar inzwischen Angebotspreise systematisch erheben, die aber bestimmte Segmente des Immobilienmarktes systematisch vernachlässigen und tendenziell die tatsächlich gezahlten Preise überschätzen.

Die vorhandene regionale Variation von Lohnniveau, Lebenshaltungskosten sowie Arbeits- und Wohnungsmarktbedingungen in Deutschland lässt sich anhand der 
Abb. 1 und 2 illustrieren. Ost-West-, Nord-Süd- und Stadt-Land-Unterschiede sind erkennbar.

Angebotsmieten und Leerstände von Wohnungen sind negativ korreliert; höhere Mieten lassen sich dort durchsetzen, wo Wohnraum knapp ist. Das Lohnniveau ist sowohl mit dem Verhältnis von offenen Stellen und Arbeitslosigkeit wie mit den Angebotsmieten positiv korreliert. Das bedeutet aber nicht, dass das Realeinkommen, d.h., die Kaufkraft des Einkommens, überall etwa gleich hoch wäre. Schon die Analyse von BBSR (2009) zeigte, dass Nominal- und Realeinkommen positiv korreliert sind, dass also höhere Mieten durch höhere Einkommen überkompensiert werden. Ein höheres Lohnniveau findet sich im Durchschnitt auf angespannten Arbeitsmärkten, auf denen im Verhältnis zu der Zahl der Arbeitslosen mehr Stellen angeboten werden. Aufgrund der positiven Verknüpfung von Lohnniveau und Angebotsmieten einerseits und der negativen Verknüpfung von Angebotsmieten und Leerständen andererseits wird jedoch ein Teil der Realeinkommensvorteile der produktiven Regionen durch längere Suchzeiten auf den Wohnungsmärkten bzw. längere Pendelwege ausgeglichen. Die dieser Argumentation zugrunde liegenden Zusammenhänge sind für die Kreise 2014 als Punktwolken und Regressionslinien in den Abb. 3 und 4 und im Anhang als (auf dem 1\%-Niveau statistisch signifikante) Korrelationskoeffizienten in Tab. 1 dargestellt.

\section{Schlussbemerkungen}

Attraktivität hat für Städte eine Kehrseite: hohe reale Wohnkosten, weites Pendeln oder lange Warteschlangen. Eine rigide Deckelung der Mieten kann nicht verhindern, dass Attraktivität nicht umsonst zu haben ist. Im Gegenteil, auf strikt regulierten Märkten, bei denen der Preismechanismus außer Kraft gesetzt wird, gedeihen Begünstigung von Insidern, Korruption und Verschwendung. Bindende Mietobergrenzen werden die Lasten umverteilen: Immobile Mieter werden zumindest kurzfristig gewinnen, mobile Outsider verdrängt. Statt Wohnraum in begehrten Lagen zu erweitern, werden umfassende und strikte Mietobergrenzen Wohnraum verknappen.

Mobile Individuen werden aufgrund inhärenter Prozesse auf interdependenten Wohnungs- und Arbeitsmärkten im Gesamtsystem der Regionen für geringere Standortattraktivität, niedrigere Löhne und längere Suchzeiten auf dem Arbeitsmarkt durch geringere Bodenpreise bzw. Mieten und schneller verfügbaren Wohnraum weitgehend kompensiert. Für wenig mobile Personen mit starker Bindung an bestimmte Regionen hingegen stellen Märkte diesen Ausgleich nicht automatisch bereit. Um für diese Gruppe Bedarfsgerechtigkeit herzustellen, bedarf es möglichst zielgenauer Unterstützung. Zunächst ist also an (Einkommens-) Transfers an betroffene Individuen zu denken. Regionenbezogene Politiken sollten solche personenbezogene Unterstützung nur dann ergänzen, wenn individuelle Transfers mit zu hohen Verzerrungen, Transaktionskosten bzw. Sickereffekten verbunden sind. Insofern ist die öffentliche Daseinsvorsorge in betroffenen Regionen allerdings ein wichtiger Baustein zur Herstellung von Bedarfsgerechtigkeit. 
Open Access Dieser Artikel wird unter der Creative Commons Namensnennung 4.0 International Lizenz (http://creativecommons.org/licenses/by/4.0/deed.de) veröffentlicht, welche die Nutzung, Vervielfältigung, Bearbeitung, Verbreitung und Wiedergabe in jeglichem Medium und Format erlaubt, sofern Sie den/die ursprünglichen Autor(en) und die Quelle ordnungsgemäß nennen, einen Link zur Creative Commons Lizenz beifügen und angeben, ob Änderungen vorgenommen wurden.

\section{Appendix}

Tab. 1 Korrelationskoeffizienten

\begin{tabular}{lcccc}
\hline Variablen & Leerstand & $\begin{array}{c}\text { Offene Stellen/ } \\
\text { Arbeitslose }\end{array}$ & Angebotsmiete & Lohnniveau \\
\hline $\begin{array}{l}\text { Leerstand } \\
\text { Offene Stellen/ }\end{array}$ & 1,000 & & & \\
Arbeitslose & $-0,286$ & 1,000 & & \\
Angebotsmiete & $-0,559$ & 0,358 & 1,000 & 1,000 \\
Lohnniveau & $-0,364$ & 0,326 & 0,679 & \\
\hline
\end{tabular}




\section{Literatur}

Ahlfeldt GM (2011) If Alonso was right: modelling accessibility and explaining the residential land gradient. J Reg Sci 51(2):318-338

Ahlfeldt GM, Maennig W (2010) Impact of sports arenas on land values: evidence from Berlin. Ann Reg Sci 44:205-227

Ahlfeldt GM, Pietrostefani E (2019) The economic effects of density: a synthesis. J Urban Econ 111:93-107

Albouy D (2009) The unequal geographic burden of federal taxation. J Polit Econ 117:635-667

Albouy D (2012) Are big cities bad places to live? Estimating quality of life across metropolitan areas. Technischer Bericht. University of Michigan, Ann Arbor

Albouy D (2016) What are cities worth? Land rents, local productivity, and the capitalization of amenity values. Rev Econ Stat 98(3):477-487

Albouy D, Lue B (2015) Driving to opportunity: local rents, wages, commuting, and sub-metropolitan quality of life. J Urban Econ 89:74-92

Bayer P, Keohane N, Timmins C (2009) Migration and hedonic valuation: the case of air quality. J Environ Econ Manage 58:1-14

BBSR (Hrsg) (2009) Regionaler Preisindex, Nummer 30, Bonn.

Büttner T, Ebertz A (2009) Quality of life in the regions: results for German counties. Ann Reg Sci 43:89-112

Dauth W, Findeisen S, Moretti E, Südekum J (2018) Matching in cities. NBER Working Paper, 25227

Duranton G, Puga D (2015) Urban land use. In: Duranton G, Henderson JV, Strange WC (Hrsg) Handbook of regional and Urban economics, Bd. 5B, Kapitel 8. Elsevier, Amsterdam, S 467-560

Ganzer A, Schmucker A, vom Berge P, Wurdack A (2016) Sample of integrated labour market biographies regional file 1975-2014 (SIAB-R 7514). FDZ data report, 01/2017 (en). Technischer Bericht. Institute for Employment Research (IAB), Nürnberg

Gyourko J, Tracy J (1991) The structure of local public finance and the quality of life. J Polit Econ 99:774-806

Han L, Strange WC (2015) The microstructure of housing markets: search, bargaining, and brokerage. In: Duranton G, Henderson JV, Strange WC (Hrsg) Handbook of regional and Urban economics, Bd. 5B, Kapitel 13. Elsevier, Amsterdam, S 813-886

Harris JR, Todaro MP (1970) Migration, unemployment and development: a two-sector analysis. Am Econ Rev 60:126-142

Mense A, Michelsen C, Kholodilin KA (2019) The effects of second-generation rent control on land values. AEA Pap \& Proc 109:385-388

Roback J (1982) Wages, rents, and the quality of life. J Polit Econ 90:1257-1278

Rosen S (1979) Wages-based indexes of Urban quality of life. In: Mieszkowski P, Straszheim M (Hrsg) Current issues in Urban economics. John Hopkins University Press, Baltimore

Winters JV (2010) Differences in quality of life estimates using rents and home values. MPRA, 24270

Wrede M (2015) Wages, rents, unemployment, and the quality of life: a consistent theory-based measure. J Reg Sci 55:609-625

Zenou Y (2009) Urban labor economics. Cambridge University Press, Cambridge 